\title{
COMPLEX INTERTWININGS AND QUANTIFICATION OF DISCRETE FREE MOTIONS
}

\author{
LAURENT MiCLO*
}

\begin{abstract}
The traditional quantification of free motions on Euclidean spaces into the Laplacian is revisited as a complex intertwining obtained through Doob transforms with respect to complex eigenvectors. This approach can be applied to free motions on finitely generated discrete Abelian groups: $\mathbb{Z}^{m}$, with $m \in \mathbb{N}$, finite tori and their products. It leads to a proposition of Markov quantification. It is a first attempt to give a probability-oriented interpretation of $\exp (\xi L)$, when $L$ is a (finite) Markov generator and $\xi$ is a complex number of modulus 1 .
\end{abstract}

Mathematics Subject Classification. 81Q35, 47D08, 35K08, 39A12, 60J27.

Received July 14, 2017. Accepted October 9, 2018.

\section{INTRODUCTION}

Broadly speaking, quantification is the mathematical link between classical and quantum mechanics and has led to the tremendous development of semi-classical limits, see e.g. the book of Zworski [6] and the references therein. Usually, the underlying state spaces and dynamics are continuous, since it is important to be able to consider high frequencies or large values in the spectrum of the associated Hamiltonian operators, quantifying the classical energies. Nevertheless, quantification has also been considered for discrete spaces, specially in the context of wave equations, see for instance Macià [3, 4], Mielke et al. [2, 5]. In general the authors also "quantify" space, by requiring that the distance separating nearest neighbors is of the same order as the semi-classical parameter $h>0$, in some sense their results concern approximation of continuous spaces. Here, we would like to consider fixed discrete state spaces, even finite sets, e.g. just on $\mathbb{Z}_{3}:=\mathbb{Z} /(3 \mathbb{Z})$. It leads us to propose a general definition of quantification in the context of Markov process theory and to see that it is meaningful, at least for the simplest examples of free motions. Of course this first attempt will have to be tested with more interesting examples, but it gives a feeling of what we are looking for.

\subsection{Definition of a Markovian quantification}

Let $V$ be a metric space, whose distance is denoted $\rho$, endowed with a (non-negative) measure $\mu$. Consider a Markov generator $L$ which is self-adjoint in $\mathbb{L}^{2}(\mu, \mathbb{C})$, in probabilistic terminology, we say that $\mu$ is reversible for $L$. Functional calculus enables one to define the operator $P_{\xi}:=\exp (\xi L)$ for any $\xi \in \mathbb{C}$. At least for $\xi \in \mathbb{C}_{+}$, the set of complex numbers whose real part is non-negative, the domain of $P_{\xi}$ is the whole space $\mathbb{L}^{2}(\mu, \mathbb{C})$, since

Keywords and phrases: Quantification, free motion, Markov process, Doob transform, intertwining.

Institut de Mathématiques de Toulouse, UMR 5219 Université de Toulouse and CNRS, Toulouse, France.

* Corresponding author: laurent.miclo@math.univ-toulouse.fr 
the spectrum of $L$ is included into $(-\infty, 0]$. When $L$ is a jump generator with bounded jump rates (in particular when $V$ is finite), the domain of $P_{\xi}$ is $\mathbb{L}^{2}(\mu, \mathbb{C})$ for all $\xi \in \mathbb{C}$.

Let $\mathcal{V}$ be another topological space endowed with a continuous and onto mapping $\pi: \mathcal{V} \rightarrow V$. It means that $\mathcal{V}$ can be seen as a weak kind of bundle over $V$ : for any $y \in V$, denote $\mathcal{V}_{y}:=\pi^{-1}(y)$, it will be convenient to write the elements of $\mathcal{V}$ under the form $(y, z)$, where $y \in V$ and $z \in \mathcal{V}_{y}$. The base component $y$ should be thought of as a position and the fiber component $z$ as a generalized speed or impulsion. A priori no assumption is made on the fibers $\mathcal{V}_{y}$, for $y \in V$, they could be not all the same. Let $\mathcal{L}$ be a Markov generator on $\mathcal{V}$ such that for any $\left(y_{0}, z_{0}\right) \in \mathcal{V}$, the martingale problem associated to $\mathcal{L}$ and to the initial point $\left(y_{0}, z_{0}\right)$ is well-posed. We will denote by $\mathcal{X}:=(\mathcal{X}(s))_{s \geqslant 0}:=(Y(s), Z(s))_{s \geqslant 0}$ a corresponding càdlàg Markov process and $\mathbb{P}_{\left(y_{0}, z_{0}\right)}$ will stand for the underlying probability measure. It is convenient to add the time as a coordinate to $\mathcal{V}:$ on $\mathfrak{V}:=\mathbb{R}_{+} \times \mathcal{V}$, whose generic elements are denoted by $(t, y, z)$, consider the generator

$$
\mathfrak{L}:=\partial_{t}+\mathcal{L}
$$

A corresponding Markov process starting from $\left(t_{0}, y_{0}, z_{0}\right)$ is $\mathfrak{X}:=(\mathfrak{X}(s))_{s \geqslant 0}:=\left(t_{0}+s, Y(s), Z(s)\right)_{s \geqslant 0}$ under $\mathbb{P}_{\left(y_{0}, z_{0}\right)}$. The latter underlying probability will also be denoted $\mathbb{P}_{\left(t_{0}, y_{0}, z_{0}\right)}$ to indicate that $\mathfrak{X}$ is starting from $\left(t_{0}, y_{0}, z_{0}\right)$ (and $\mathbb{P}_{\left(y_{0}, z_{0}\right)}$ will stand for $\left.\mathbb{P}_{\left(0, y_{0}, z_{0}\right)}\right)$.

We say that $L$ is a quantification of $\mathcal{L}$ if there exists a family $\left(F_{h}\right)_{h>0}$ of continuous and bounded mappings from $\mathfrak{V} \times V$ to $\mathbb{C}$ satisfying the three following conditions:

(H1) Probability density: for any $h>0$ and any $(t, y, z) \in \mathfrak{V}$, we have

$$
\int_{V}\left|F_{h}(t, y, z ; x)\right|^{2} \mu(d x)=1
$$

(H2) Concentration: for any $r>0$ and any compact set $K \subset \mathbb{R}_{+} \times V$,

$$
\lim _{h \rightarrow 0_{+}} \frac{1}{h} \sup _{(t, y, z) \in \mathfrak{V}:(t, y) \in K} \int_{\rho(x, y) \geqslant r}\left|F_{h}(h t, y, z ; x)\right|^{2} \mu(d x)=0 .
$$

(H3) Intertwining: for any $\left(y_{0}, z_{0}\right) \in \mathcal{V}$, there exists $\xi_{0} \in \mathbb{T}$, the circle of complex numbers of modulus 1 , such that,

$$
\forall h>0, \forall s \geqslant 0, \forall x \in V, \quad \mathbb{E}_{\left(0, y_{0}, z_{0}\right)}\left[F_{h}(\mathfrak{X}(s) ; x)\right]=P_{\xi_{0} s}\left[F_{h}\left(0, y_{0}, z_{0} ; \cdot\right)\right](x) .
$$

Note that the rhs is a priori only defined $\mu$-a.s. in $x \in V$, since $P_{\xi_{0} h s}$ is an operator on $\mathbb{L}^{2}(\mu, \mathbb{C})$. Nevertheless, as an immediate consequence of dominated convergence, the lhs is continuous with respect to $x \in V$. The meaning of the above equality is thus that there exists a continuous version of $P_{\xi_{0} s}\left[F_{h}\left(0, y_{0}, z_{0} ; \cdot\right)\right]$ given by the lhs. In all our examples of discrete free motions, $\xi_{0}$ will in fact only be dependent on $z_{0}$.

A drawback of Assumption (H3) is its lack of stability by tensorization (except in the traditional situation where $\xi_{0}$ does not depend on $\left.\left(y_{0}, z_{0}\right) \in \mathcal{V}\right)$ and the following extension shall more generally be considered. We say that a commuting family $\left(L^{(j)}\right)_{j \in J}$ of reversible Markov generators in $\mathbb{L}^{2}(\mu, \mathbb{C})$, where $J$ is a finite index set, is a multi-dimensional quantification of $\mathcal{L}$, when there exists a family $\left(F_{h}\right)_{h>0}$ of mappings as above satisfying (H1), (H2) and

(H4) Multi-dimensional intertwining: for any $\left(y_{0}, z_{0}\right) \in \mathcal{V}$, there exists a family $\left(\xi_{0}^{(j)}\right)_{j \in J} \in \mathbb{T}^{J}$, such that,

$$
\forall h>0, \forall s \geqslant 0, \forall x \in V, \quad \mathbb{E}_{\left(0, y_{0}, z_{0}\right)}\left[F_{h}(\mathfrak{X}(s) ; x)\right]=\exp \left(s \sum_{j \in J} \xi_{0}^{(j)} L^{(j)}\right)\left[F_{h}\left(0, y_{0}, z_{0} ; \cdot\right)\right](x) .
$$


Let us make a few comments about the definition of quantification by (H1), (H2) and (H3). The multidimensional extension will be justified by Theorem 1.8 below and discussed at the end of Section 5 .

Remark 1.1. In the usual real free motion Schrödinger case recalled in Theorem 1.5 below, $\xi_{0}=i$ (or $\xi_{0}=-i$ ) does not depend on $\left(y_{0}, z_{0}\right) \in \mathcal{V}$. Under natural assumptions, it is equivalent to the fact that for any $h>0$ and $x \in V$, the process $\left(F_{h}(\mathcal{X}(s) ; x)\right)_{s \geqslant 0}$ is deterministic. Indeed, for the direct implication, note that $P_{\xi_{0}}$ is a unitary operator for any $\xi_{0} \in i \mathbb{R}$, so we get for all $h>0,\left(y_{0}, z_{0}\right) \in \mathcal{V}$ and $s \geqslant 0$,

$$
\begin{aligned}
\int\left|P_{\xi_{0} h s}\left[F_{h}\left(0, y_{0}, z_{0} ; \cdot\right)\right](x)\right|^{2} \mu(d x) & =\int_{1}\left|F_{h}\left(0, y_{0}, z_{0} ; x\right)\right|^{2} \mu(d x) \\
& =1
\end{aligned}
$$

where we used (H1).

On the other hand, by Cauchy-Schwarz' inequality, we have for any $h>0,\left(y_{0}, z_{0}\right) \in \mathcal{V}, s \geqslant 0$ and $x \in V$,

$$
\left|\mathbb{E}_{\left(y_{0}, z_{0}\right)}\left[F_{h}(\mathfrak{X}(h s) ; x)\right]\right|^{2} \leqslant \mathbb{E}_{\left(y_{0}, z_{0}\right)}\left[\left|F_{h}(\mathfrak{X}(h s) ; x)\right|^{2}\right],
$$

so that

$$
\begin{aligned}
\int\left|\mathbb{E}_{\left(y_{0}, z_{0}\right)}\left[F_{h}(\mathfrak{X}(h s) ; x)\right]\right|^{2} \mu(d x) & \leqslant \int \mathbb{E}_{\left(y_{0}, z_{0}\right)}\left[\left|F_{h}(\mathfrak{X}(h s) ; x)\right|^{2}\right] \mu(d x) \\
& =\mathbb{E}_{\left(y_{0}, z_{0}\right)}\left[\int\left|F_{h}(\mathfrak{X}(h s) ; x)\right|^{2} \mu(d x)\right] \\
& =\mathbb{E}_{\left(y_{0}, z_{0}\right)}[1] \\
& =1 .
\end{aligned}
$$

Furthermore, assumption (H3) implies that

$$
\forall h>0, \forall\left(y_{0}, z_{0}\right) \in \mathcal{V}, \forall s \geqslant 0, \forall x \in V, \quad\left|\mathbb{E}_{\left(y_{0}, z_{0}\right)}\left[F_{h}(\mathfrak{X}(h s) ; x)\right]\right|^{2}=\left|P_{\xi_{0} h s}\left[F_{h}\left(0, y_{0}, z_{0} ; \cdot\right)\right](x)\right|^{2} .
$$

It follows from the above computations that we must have equality in the Cauchy-Schwarz' inequality (1.2). This is only possible if $F_{h}(\mathfrak{X}(h s) ; x)$ is a deterministic quantity under $\mathbb{P}_{\left(y_{0}, z_{0}\right)}$, for any fixed $h>0,\left(y_{0}, z_{0}\right) \in \mathcal{V}$, $s \geqslant 0$ and $x \in V$. We can go further and conclude that the process $\mathcal{X}$ itself is deterministic, under the assumption that the family $\left(F_{h}\right)_{h>0}$ is discriminating, in the sense that for any $(t, y, z) \neq\left(t, y^{\prime}, z^{\prime}\right) \in(0,+\infty) \times \mathcal{V}$, there exist $h>0$ and $x \in V$ such that $F_{h}(t, y, z ; x) \neq F_{h}\left(t, y^{\prime}, z^{\prime} ; x\right)$ (this condition will be satisfied in all our examples).

Conversely, when $\left(F_{h}(\mathcal{X}(s) ; x)\right)_{s \geqslant 0}$ is deterministic, we get in (H3)

$$
\forall h>0, \forall\left(y_{0}, z_{0}\right) \in \mathcal{V}, \forall s \geqslant 0, \forall x \in V, \quad F_{h}(\mathfrak{X}(h s) ; x)=P_{\xi_{0} h s}\left[F_{h}\left(0, y_{0}, z_{0} ; \cdot\right)\right](x) .
$$

Taking the square of the modulus and integrating, we deduce that

$$
\begin{aligned}
\int\left|P_{\xi_{0} h s}\left[F_{h}\left(0, y_{0}, z_{0} ; \cdot\right)\right](x)\right|^{2} \mu(d x) & =1 \\
& =\int\left|F_{h}\left(0, y_{0}, z_{0} ; x\right)\right|^{2} \mu(d x) .
\end{aligned}
$$

Now assume that $L$ is irreducible, namely its eigenvalue 0 is of multiplicity 1 . Its eigenspace is then reduced to the constant functions. Spectral calculus implies that for $\xi_{0} \notin i \mathbb{R}$ and $s, h>0$, all the eigenvalues, except 1 , of $P_{\xi_{0} h s}$ have a modulus smaller (respectively larger) than 1 if $\Re\left(\xi_{0}\right)>0$ (resp. $\Re\left(\xi_{0}\right)<0$ ). Thus for the 
above equality to hold, the mapping $V \ni x \mapsto F_{h}\left(0, y_{0}, z_{0} ; x\right)$ must be constant, for any $h>0$ and $\left(y_{0}, z_{0}\right) \in \mathcal{V}$. Assumption (H2) then leads to a contradiction, except in the trivial case where $V$ is a singleton. Thus, we must have $\xi_{0} \in(i \mathbb{R}) \cap \mathbb{T}=\{ \pm i\}$.

These considerations show why when $V$ is discrete (then $\mathcal{X}$ cannot be deterministic if we want it to be Markovian), one has to consider for $\xi_{0}$ other elements of $\mathbb{T}$ than $\pm i$. The fact that we imposed $\xi_{0} \in \mathbb{T}$ is just a normalization taking into account that it is always possible to multiply a Markov generator by a positive constant: it amounts to multiply the time by the same constant.

Remark 1.2. Let us discuss the meaning of the above quantification, in a very heuristic way. According to (H1) and (H2), for any $s \geqslant 0$ and $x \in V$, we have for $h>0$ small

$$
\begin{aligned}
\left|F_{h}(\mathfrak{X}(h s), x)\right|^{2} & =\left|F_{h}(h s, Y(h s), Z(h s) ; x)\right|^{2} \\
& \approx \delta_{Y(h s)}(x),
\end{aligned}
$$

and

$$
\left|F_{h}\left(0, y_{0}, z_{0} ; \cdot\right)\right|^{2} \approx \delta_{y_{0}}
$$

where for any $y \in V, \delta_{y}$ stands for the Dirac mass at $y$. Taking into account the previous remark, we "infer", in the case where $\xi_{0}=i$, that (H3) writes

$$
\delta_{Y(h s)}(x) \approx\left|P_{i h s}\left[\sqrt{\delta_{y_{0}}}\right](x)\right|^{2}
$$

or

$$
\sqrt{\delta_{Y(h s)}}(x) \approx\left|P_{i h s}\left[\sqrt{\delta_{y_{0}}}\right](x)\right|
$$

namely, $\left|P_{i h s}[\cdot]\right|$ has approximatively transported $\sqrt{\delta_{y_{0}}}$ into $\sqrt{\delta_{Y(h s)}}$, if a sense could be given to these expressions. More rigorously, an equality cannot hold in (1.3), since we would deduce that the evolution of $Y(h s)$, for $s \geqslant 0$, does not depend on $z_{0}$, which is typically not true. In fact, an important motivation for the quantification procedure is to remove the fiber components of the evolution of $(Y(h s), Z(h s))_{s \geqslant 0}$ and to include them (as "phases") into the evolving complex-valued distributions on $V$ given by the family $\left(P_{i h s}\left[F_{h}\left(0, y_{0}, z_{0} ; \cdot\right)\right]\right)_{s \geqslant 0}$. According to the interpretation of $y$ and $z$ as position and speed components, the stochastic process $(Y(h s))_{s \geqslant 0}$ could be seen as a second order Markov process (while $(Y(h s), Z(h s))_{s \geqslant 0}$ is a usual, i.e. first order, Markov process). Quantification then looks like a crazy attempt to approximate the second order Markov process $(Y(h s))_{s \geqslant 0}$ by a "first order Markov process" on $V$ whose semi-group would be $\left(P_{i h s}\right)_{s \geqslant 0}$, puzzling as the imaginary-valued times remain. A similar heuristic could be proposed in the case of discrete state spaces $V$ : then for all $y \in V$, $\sqrt{\delta_{y}}=\delta_{y}$, interpreting the Dirac mass as a density with respect to the counting measure (i.e. the Kronecker delta), and (1.3) would have to be modified into the statement $\mathbb{E}\left[\exp (i \Theta(h s)) \delta_{Y(h s)}\right] \approx P_{\xi_{0} h s}\left[\delta_{y_{0}}\right]$, where $\Theta(h s)$ is a random real phase, with $\Theta(0)=0$. A difference with the usual Schrödinger case is that a part of the information about the fiber component will be transferred to the complex time, since $\xi_{0}$ will depend on $z_{0}$.

Remark 1.3. The concentration property of (H2) is of order 1 , in the sense that a concentration of order $k \in \mathbb{Z}_{+}$can be defined by requiring that for any $r>0$ and any compact set $K \subset \mathbb{R}_{+} \times V$,

$$
\lim _{h \rightarrow 0_{+}} \frac{1}{h^{k}} \sup _{(t, y, z) \in \mathfrak{V}:(t, y) \in K} \int_{\rho(x, y) \geqslant r}\left|F_{h}(h t, y, z ; x)\right|^{2} \mu(d x)=0
$$

In the usual Schrödinger case, the concentration will even be strong, in the sense that it is of any order $k \in \mathbb{Z}_{+}$. 
The order 1 is the smallest meaningful order for quantification: for fixed $s>0$ and small $h$, the movement of $\mathcal{X}(h s)$ from $\mathcal{X}(0)$ will typically be of order $h$ (either in distance or in probability to have jumped to a distance of order 1), because we are looking for generators $\mathcal{L}$ leading to "ballistic" behaviors, even if the associated motions are not deterministic. As a consequence, to get a pertinent result, the concentration should be stronger than $h$ and this is exactly what is requiring (H2). In our simple discrete examples, we will have for $h>0$ small, with the notations of (H2),

$$
\sup _{(t, y, z) \in \mathfrak{V}:(t, y) \in K} \int_{\rho(x, y) \geqslant r}\left|F_{h}(h t, y, z ; x)\right|^{2} \mu(d x)=\mathcal{O}\left(h^{2}\right) .
$$

Remark 1.4. The above definition of quantification is not completely satisfactory, because a Markov process on $V$ can be seen as its own quantification. Indeed, let be given a jump generator $L$ on a discrete space $V$. It is the quantification of $\mathcal{L}:=L$ on $\mathcal{V}:=V \times\{0\} \equiv V$, by choosing $\xi_{0}=1$ and

$$
\forall h>0, \forall(t, y, 0) \in \mathfrak{V}, \forall x \in V, \quad F_{h}(t, y, 0 ; x):=\delta_{y, x}
$$

where the rhs is the Kronecker delta. A similar result holds on general state spaces $V$ and Markov generators $L$, by considering a family $\left(F_{h}\right)_{h>0}$ such that $\left|F_{h}(t, y, 0 ; x)\right|^{2} \mu(d x)$ is an approximation of the Dirac mass at $y$ and $F_{h}(t, y, 0 ; x)$ is symmetrical in $y, x$ and does not depend on $t$.

This kind of degeneracy could be avoided by requiring in (H3) that $\xi_{0} \in \mathbb{T} \backslash\{-1,1\}$, the case $\xi=-1$ appearing for instance when $L$ generates deterministic motions that can be reversed in time. This prohibition of real values for $\xi_{0}$ is in the spirit of Remark 1.2, where we are trying to get a probabilistic interpretation of $P_{\xi_{0} t}$, for $t>0$, task which is particularly puzzling when $\xi_{0} \notin \mathbb{R}$. Furthermore, we are looking for generators $\mathcal{L}$ whose associated motions contain the smallest possible quantity of randomness (the most ballistic, with the wording of Rem. 1.3) and according to Remark 1.1, this is reflected by the least real possible $\xi_{0}$. Nevertheless, it will become apparent in Sections 4 and 5 that real values should be allowed for $\xi_{0}$ for some $\left(y_{0}, z_{0}\right) \in \mathcal{V}$.

Another drawback of the present definition of quantification, is that given the Markov generator $L$, there is not a unique $\mathcal{L}$ to which $L$ is the quantification, up to natural identifications. Indeed, given a quantification, another one can be obtained by enlarging the fibers (for instance by duplicating them).

A notion of minimal quantification up to appropriate isomorphisms is thus missing and this minimization should concern first the real part of $\xi_{0}$ and next the size of the fibers of $\mathcal{V}$.

In the present paper, we will investigate some simple situations of free motions: the generator $\mathcal{L}$ will only act on the base component $y$, but in a way parametrized by the fiber component $z$. Thus the fiber component will not move: for any $s \geqslant 0, Z(s)=z_{0}$. This feature imposes that all the fibers are the same, property which was not required in the general definition given above. We will have that $\mathcal{V}=V \times W$, where $W$ is another topological space, even if its topology will not play a role when the fiber component does not move.

As already mentioned, our main goal is to define quantification on finite state spaces $V$. This demand is the reason why we allow the process $(\mathcal{X}(s))_{s \geqslant 0}$ to be stochastic and for the complex number $\xi_{0} \in \mathbb{T}$ not to be necessary equal to $\pm i$, in accordance with Remark 1.1. In fact our initial motivation is an inverse problem: given a finite irreducible Markov generator $L$, we are wondering if we can find a "natural" dynamics to which it is a quantification. It leads to other questions that are out of the scope of this paper, in particular because the limitations pointed out in Remark 1.4 have to be overcome first: is there always a "semi-classical limit" (i.e. a corresponding minimal generator $\mathcal{L}$ ), is it unique? These interrogations will be interesting even for non-reversible generators, in fact, the reversibility assumption was mainly adopted to simplify the definition of the operators $\exp (\xi L)$, for $\xi \in \mathbb{C}$, but there is no such difficulty when $V$ is finite. Here we will answer the first question only for the usual Laplacian on a discrete multidimensional torus $V=\mathbb{Z}_{n_{1}} \times \mathbb{Z}_{n_{2}} \times \cdots \times \mathbb{Z}_{n_{m}}$, where $m \in \mathbb{N}$ and $n_{1}, n_{2}, \ldots, n_{m} \in \mathbb{N} \backslash\{1\}$. In future works, we hope to deal with the challenges of adding potential energy terms or of considering general weighted graphs. This is not just for the sake of generality: to define (stochastic) 
Hamiltonian dynamics on graphs whose quantification corresponds to Metropolis algorithms is an interesting perspective in the field of optimizing and sampling stochastic algorithms.

\subsection{Results on free motions}

After recalling the classical example of the free motion on $\mathbb{R}$, we will present the quantification of discrete free motions, on $\mathbb{Z}$ and on finite tori.

For the free motion on $\mathbb{R}$, we take $V:=\mathbb{R}$ endowed with the Laplacian $L=\partial^{2}$. The fiber space $W$ is also equal to $\mathbb{R}$ and we consider the operator $\mathcal{L}=2 z \partial_{y}$ acting on $V \times W=\mathbb{R}^{2}$. We have

Theorem 1.5. The generator $L$ is a quantification of $\mathcal{L}$ and in (H3), $\xi_{0}=i$ is independent of $\left(y_{0}, z_{0}\right)$.

Here is our first example of quantification of a discrete free motion. Take $V:=\mathbb{Z}$ endowed with the generator $L$ acting on bounded functions $f$ on $\mathbb{Z}$ via

$$
\forall x \in \mathbb{Z}, \quad L[f](x):=f(x+1)+f(x-1)-2 f(x) .
$$

The fiber space is $W=[-\pi, \pi)$ and we consider the operator $\mathcal{L}$ acting on bounded functions $f$ defined on $\mathbb{Z} \times[-\pi, \pi)$ by

$$
\forall(y, z) \in \mathbb{Z} \times[-\pi, \pi), \quad \mathcal{L}[f](y, z):=2|\sin (z)|(f(y+\operatorname{sign}(z), z)-f(y, z),
$$

where $\operatorname{sign}(z):=1$ when $z \geqslant 0$ and $\operatorname{sign}(z)=-1$ when $z<0$.

We have the following theorem.

Theorem 1.6. The generator $L$ is a quantification of $\mathcal{L}$ and in $(H 3), \xi_{0}=i \exp (-i|z|)$.

Our second example of quantification of a discrete free motion is on the finite circle $\mathbb{Z}_{n}$, where $n \in \mathbb{N} \backslash\{1\}$. Consider on $\mathbb{Z}_{n}$ the discrete Laplacian $L$, equally given by (1.4) for $x \in \mathbb{Z}_{n}$. The fiber space is now

$$
W_{n}:=\frac{2 \pi}{n} \llbracket 0, n-1 \rrbracket .
$$

and we consider the operator $\mathcal{L}$ acting on any function $f$ defined on the finite set $\mathbb{Z}_{n} \times W_{n}$ via (1.5), where $(y, z) \in \mathbb{Z}_{n} \times W_{n}$. In this context, Theorem 1.6 is valid, apparently without amendment, but the domains of the operators are not the same.

Theorem 1.7. The generator $L$ is a quantification of $\mathcal{L}$ and in $(H 3), \xi_{0}=i \exp (-i|z|)$.

To see the interest of (H4), we now come to a "multi-dimensional" example. We take $V=\mathbb{Z}_{n_{1}} \times \mathbb{Z}_{n_{2}} \times \cdots \times$ $\mathbb{Z}_{n_{m}}$, where $m \in \mathbb{N}$ and $n_{1}, n_{2}, \ldots, n_{m} \in \mathbb{N} \backslash\{1\}$. Let $J:=\llbracket m \rrbracket:=\{1,2, \ldots, m\}$ and for $j \in J$, consider the generator $L^{(j)}$ acting on functions $f$ defined on $V$ via

$$
\forall x \in V, \quad L^{(j)}[f](x):=f\left(x+e_{j}\right)+f\left(x-e_{j}\right)-2 f(x),
$$

where $e_{j}$ is the element of $V$ whose coordinates are all 0 , except the $j$-th one equal to $1 \in \mathbb{Z}_{n_{j}}$. Let $W=$ $W_{n_{1}} \times W_{n_{2}} \times \cdots \times W_{n_{m}}$, where the factor spaces are defined as in (1.6). Consider the operator $\mathcal{L}$ acting on functions $f$ defined on $V \times W$ via

$$
\forall(y, z) \in V \times W, \quad \mathcal{L}[f](y, z):=2 \sum_{j \in J}\left|\sin \left(z_{j}\right)\right|\left(f\left(y+\operatorname{sign}\left(z_{j}\right), z\right)-f(y, z),\right.
$$

where $\left(z_{1}, z_{2}, \ldots, z_{m}\right)$ are the coordinates of a generic element $z \in W$.

The following result can be deduced from Theorem 1.7 by tensorization. 
Theorem 1.8. The family of generators $\left(L^{(j)}\right)_{j \in J}$ is a multi-dimensional quantification of $\mathcal{L}$ and in (H4), $\xi_{0}^{(j)}=i \exp \left(-i\left|z_{j}\right|\right)$, for all $j \in J$.

All these results will be in proven in the same manner, via an intertwining using complex kernels. It will also be applied to the free motion on the circle $V$, seen as $\mathbb{R} /(2 \pi \mathbb{Z})$, endowed with the Laplacian $L=\partial^{2}$. The fiber space is now $\mathbb{Z}$ and we consider the operator $\mathcal{L}=2 z \partial_{y}$ acting on $V \times W=\mathbb{R} /(2 \pi \mathbb{Z}) \times \mathbb{Z}$. Theorem 1.5 is valid in this context:

Theorem 1.9. The generator $L$ is a quantification of $\mathcal{L}$ and in (H3), $\xi_{0}=i$ is independent of $\left(y_{0}, z_{0}\right)$.

The discreteness of the fiber space $\mathbb{Z}$ is now quite suspect and we are wondering if Theorem 1.9 is true with the same operator $\mathcal{L}$ but with $W=\mathbb{R}(V \times W$ should then be seen as the cotangent space of the circle). If it is not, it would mean that our definition of quantification is too strict: in (H3) and (H4), the equality should be required only up to additive terms negligible with respect to $h$, as in (H2). In the present paper, we fostered the investigation of the perfect intertwinings (H3) and (H4), valid for all times.

The plan of the paper is as follows. The next section presents our intertwining method: it is based on a Doob transform with respect to complex valued eigenvectors which do not vanish. Sections 3 and 4 treat of the free motions on $\mathbb{R}$ and $\mathbb{Z}$, respectively. The last section deal with the cases of tori. We will also see that there is no difficulty in tensorizing Theorems 1.5 and 1.9 and that the tensorization of Theorem 1.6 can be done as in Theorem 1.8.

\section{An INTERTWINING RELATION}

Here, the intertwining relation at the base of the construction of the family $\left(F_{h}\right)_{h>0}$ is deduced via a Doob transformation applied with complex-valued eigenfunctions, contrary to the usual ground state transforms, which are usually considered relatively to positive eigenvectors. The framework is a little more general than in Section 1.1, since reversibility is not required, nor even invariance with respect to a measure $\mu$.

On a state space $V$, consider a Markov generator $L \neq 0$ defined as an endomorphism on a unitary algebra $A$ of $\mathbb{C}$-valued functions. Let $\varphi \in A$ be an eigenvector of $L$ associated to an eigenvalue $\lambda \in \mathbb{C}$ (in the applications of the following sections, we will only consider reversible $L$ with real eigenvalues, nevertheless, it will be convenient to deal with $\mathbb{C}$-valued functions). Assume that $\varphi$ does not vanish on $V$ and that $\varphi^{-1} \in A$, to be able to consider the Doob transform of $L$ by $\varphi$, acting on $A$ :

$$
\widetilde{L} \cdot:=\frac{1}{\varphi} L[\varphi \cdot]-\lambda \cdot
$$

Assume that we can find $\chi, \zeta \in \mathbb{T}$ (the circle of complex numbers of modulus 1) and a Markov generator $\widehat{L}: A \rightarrow A$, such that

$$
\widetilde{L}=\chi L+\zeta \widehat{L}
$$

Remark 2.1. In general such a decomposition is not unique, as we will see in Remarks 3.1 and 4.1 of the next sections.

Suppose furthermore that $L$ and $\widehat{L}$ commute. This assumption is very strong, but will be satisfied by our free motion examples. In more general situations, as those mentioned at the end of Section 1.1, some commutation relations will enter into play, we hope to investigate them in future works.

In the introduction, $P_{\eta}$ stood for $\exp (\eta L)$ for $\eta \in \mathbb{C}$ and we would like to define similarly $\widetilde{P}_{\eta}$ and $\widehat{P}_{\eta}$. In the present framework, the meaning of the exponential is not so clear. Not wanting to obscure the simplicity of the following arguments, let us first assume that $A$ is a Banach algebra and that $L$ is a bounded operator on $A$. With these hypotheses, $P_{\eta}, \widetilde{P}_{\eta}$ and $\widehat{P}_{\eta}$ are naturally defined as exponentials for any $\eta \in \mathbb{C}$. These assumptions hold 
when $V$ is a finite set and $A$ is the space of all $\mathbb{C}$-valued functions on $V$. For a more general set of hypotheses, see Remark 2.4. The interest of the previous operators is:

Lemma 2.2. For any $\eta, \eta^{\prime} \in \mathbb{C}$, we have the intertwining relation

$$
\widehat{P}_{\eta^{\prime}} \widetilde{P}_{-\bar{\zeta}\left(\eta+\eta^{\prime}\right)}=\widetilde{P}_{-\bar{\zeta} \eta} P_{\xi \eta^{\prime}},
$$

where $\xi:=-\bar{\zeta} \chi$.

Proof. Since $\widetilde{L}=\chi L+\zeta \widehat{L}$, and $L$ and $\widehat{L}$ commute, we have

$$
\forall \eta \in \mathbb{C}, \quad \widetilde{P}_{\eta}=\widehat{P}_{\zeta \eta} P_{\chi \eta} .
$$

It follows that for any $\eta, \eta^{\prime} \in \mathbb{C}$

$$
\begin{aligned}
\widehat{P}_{\eta^{\prime}} \widetilde{P}_{-\bar{\zeta}\left(\eta+\eta^{\prime}\right)} & =\widehat{P}_{\eta^{\prime}} \widehat{P}_{-\left(\eta+\eta^{\prime}\right)} P_{\xi\left(\eta+\eta^{\prime}\right)} \\
& =\widehat{P}_{-\eta} P_{\xi \eta} P_{\xi \eta^{\prime}} \\
& =\widetilde{P}_{-\bar{\zeta} \eta} P_{\xi \eta^{\prime}}
\end{aligned}
$$

The above constructions depend on the choice of $\varphi$, but only up to a factor and it is tempting to parametrize them by the eigenvalue $\lambda$. Its multiplicity, as well as the possible choices of $\chi, \zeta$ and $\widehat{L}$ mentioned in Remark 2.1, has to be taken into account. It leads us to consider $W$ a parametrization of a multiset of eigenvalues of $L$ to which we can associate non-vanishing eigenvectors $\varphi$ as above (it seems preferable for the corresponding eigenvectors to be independent in case of multiplicity). As in the introduction, define $\mathfrak{V}:=\mathbb{R}_{+} \times V \times W$, whose generic elements are denoted $(t, y, z)$. By definition, to each $z \in W$, we associate an eigenvector $\varphi_{z}$, an eigenvalue $\lambda_{z}$, but also the complex numbers $\chi_{z}, \zeta_{z}, \xi_{z}$, the generator $\hat{L}_{z}$, etc. Consider the Markovian generator $\mathfrak{L}$ given on $\mathfrak{A}$, the unitary algebra of mappings on $\mathfrak{V}$ which are $\mathcal{C}^{1}$ in $t$ and belong to $A$ as function of $y$, by

$$
\mathfrak{L}:=\partial_{t}+\widehat{L}_{z}
$$

In this formula, the variable $z$ enters in the definition of $\widehat{L}_{z}$, which is acting on $y$. Note that the variable $z$ is not modified by the dynamic generated by $\mathfrak{L}$. With the notations of the introduction, the generator $\mathcal{L}$ is just $\widehat{L}_{z}$, so that (1.1) is valid.

The algebra $\mathfrak{A}$ is not preserved by $\mathfrak{L}$, anyway, for any $s \geqslant 0$, there is no difficulty to define $\mathfrak{P}_{s}:=\exp (s \mathfrak{L})$ on $\mathfrak{A}$ directly via

$$
\forall F \in \mathfrak{A}, \forall(t, y, z) \in \mathfrak{V}, \quad \mathfrak{P}_{s}[F](t, y, z)=\widehat{P}_{z, s}[F(t+s, \cdot, z)](y),
$$

where $\widehat{P}_{z, s}:=\exp \left(s \widehat{L}_{z}\right)$, similar notations will be used below.

Define the operator $R$ from $\mathfrak{V}$ to $V$ through

$$
\begin{aligned}
\forall(t, y, z) \in \mathfrak{V}, \forall f \in A, \quad R[f](t, y, z) & :=\widetilde{P}_{z,-\bar{\zeta}_{z} t}[f](y) \\
& =\frac{\exp \left(\lambda_{z} \bar{\zeta}_{z} t\right)}{\varphi_{z}(y)} P_{-\bar{\zeta}_{z} t}\left[\varphi_{z} f\right](y),
\end{aligned}
$$


where we used (2.1), which implies that

$$
\forall \eta \in \mathbb{C}, \quad \widetilde{P}_{z, \eta}[\cdot]=\frac{\exp \left(-\eta \lambda_{z}\right)}{\varphi_{z}} P_{\eta}\left[\varphi_{z} \cdot\right]
$$

Lemma 2.2 can be partially rewritten under the form.

Lemma 2.3. We have

$$
\forall s \in \mathbb{R}_{+}, \quad \mathfrak{P}_{s} R=R P_{\xi s} .
$$

This relation is equivalent to the generator intertwining

$$
\mathfrak{L} R=\xi R L .
$$

Proof. Consider a test function $F \in \mathfrak{A}$. It follows from (2.4) that for any test function $f$ on $\mathfrak{V}$, any $s \in \mathbb{R}_{+}$and any $(t, y, z) \in \mathfrak{V}$, we have

$$
\begin{aligned}
\mathfrak{P}_{s} R[f](t, y, z) & =\widehat{P}_{z, s}[R[f](t+s, \cdot, z)](y) \\
& =\widehat{P}_{z, s}\left[\widetilde{P}_{z,-\bar{\zeta}_{z}(t+s)}[f]\right](y) \\
& =\left(\widehat{P}_{z, s} \widetilde{P}_{z,-\bar{\zeta}_{z}(t+s)}\right)[f](y) .
\end{aligned}
$$

On the other hand, we compute that

$$
\begin{aligned}
R P_{\xi s}[f](t, y, z) & =\widetilde{P}_{z,-\bar{\zeta}_{z} t}\left[P_{\xi s}[f]\right](y) \\
& =\left(\widetilde{P}_{z,-\bar{\zeta}_{z} t} P_{\xi s}\right)[f](y),
\end{aligned}
$$

so the first announced equality is a direct consequence of Lemma 2.2.

The second equality is obtained by differentiation with respect to $s$ at $0_{+}$. Conversely, the first equality is recovered from the second one by integration.

Remark 2.4. The assumptions made before Lemma 2.2 are too strong, e.g. to deal with the classical free motion Schrödinger equation, but they can be relaxed at the expense of further notations. Let $B$ be a Banach space of functions defined on $V$ and containing $A \cap B$ as a dense subset. Let $C \subset \mathbb{C}$ be a cone containing $\mathbb{R}_{+}$ and $\left(P_{\eta}\right)_{\eta \in C}$ be a family of continuous operators on $B$ such that for any $\eta \in C,\left(P_{s \eta}\right)_{s \geqslant 0}$ is the semi-group associated to the pregenerator $\eta L$ (acting on $A \cap B$ ), in the sense of Hille-Yoshida. Make the same hypotheses for $\widetilde{L}$ and $\widehat{L}$, to get $\widetilde{B}, \widehat{B}, \widetilde{C}, \widehat{C},\left(\widetilde{P}_{\eta}\right)_{\eta \in \widetilde{C}}$ and $\left(\widehat{P}_{\eta}\right)_{\eta \in \hat{C}}$. Assume that $B=\widetilde{B}=\widehat{B}, C=\widetilde{C}=\chi C, \widehat{C} \subset C$ and $-\bar{\zeta} \widehat{C} \subset C$. Then Lemmas 2.2 and 2.3 are satisfied up to appropriate quantifications, for instance the statement of Lemma 2.2 requires $\eta, \eta^{\prime} \in \widehat{C}$.

The operator $R$ will be important to construct the family of functions $\left(F_{h}\right)_{h>0}$ presented in the introduction. More precisely, for any $h>0$ and $(y, z) \in V \times W$, we will find an appropriate function $f_{h, y, z} \in A$, concentrating around $y_{0}$ for $h>0$ small and $F_{h}$ will be defined by

$$
\forall(t, y, z) \in \mathfrak{V}, \forall x \in V, \quad F_{h}(t, y, z ; x):=R\left[f_{h, y, z}\right](t, x, z) .
$$

Before investigating more precisely these functions in the examples of the following sections, let us come back to the decomposition (2.2). The carré du champs $\Gamma$ associated to $L$ is the bilinear functional on $A \times A$ defined 
by

$$
\forall f, g \in A, \quad \Gamma[f, g]:=L[f g]-f L[g]-g L[f]
$$

We compute that for any $f \in A$,

$$
\begin{aligned}
\widetilde{L}[f] & =\frac{1}{\varphi}(L[\varphi f]-\lambda \varphi f) \\
& =\frac{1}{\varphi}(f L[\varphi]+\varphi L[f]+\Gamma[\varphi, f]-\lambda \varphi f) \\
& =L[f]+\check{L}[f]
\end{aligned}
$$

where

$$
\check{L}[\cdot]:=\frac{1}{\varphi} \Gamma[\varphi, \cdot]
$$

Thus the decomposition (2.2) is equivalent to

$$
\check{L}=(\chi-1) L+\zeta \widehat{L}
$$

This alternative writing is particularly important when $L$ is a diffusion generator. A Markov generator $L$ is of diffusion type when $A$ is stable by composition with smooth mappings $F: \mathbb{R} \rightarrow \mathbb{R}$ and that

$$
\forall f \in A, \quad L[F \circ f]=F^{\prime}(f) L[f]+\frac{F^{\prime \prime}(f)}{2} \Gamma[f, f]
$$

In this context, recall that an operator $K$ defined on $A$ is a derivation when

$$
\forall f \in A, \quad K[F \circ f]=F^{\prime}(f) K[f]
$$

The following result is well-known, see e.g. the book of Bakry, Gentil and Ledoux [1]:

Proposition 2.5. When $L$ is a diffusion generator, $\breve{L}$ is a derivation operator.

Assume now that $V$ is a differential manifold and that $A$ is the space of smooth functions. A Markov generator $L: A \rightarrow A$ is of diffusion type if and only if it is a second order operator without zero order term. When the second order part of $L$ does not vanish identically (which is just asking for $L$ not being a derivation), we deduce from Proposition 2.5 that it is natural to ask for $\chi=1$ in (2.7), if we don't want $\widehat{L}$ to contain the same second order terms as $L$, up to a factor. A derivation operator $K$ comes from a vector field if and only if $K$ transforms $\mathbb{R}$-valued functions into $\mathbb{R}$-valued functions. Furthermore this condition is equivalent to the fact that $K$ is a Markov generator (leading to the deterministic dynamical system obtained by following the corresponding vector field). Thus in this context, the existence of the decomposition (2.2) is equivalent to the existence of $\zeta \in \mathbb{T}$ such that $\bar{\zeta} \breve{L}$ comes from a vector field. When this is satisfied, we can take $\chi=1$ and $\widehat{L}=\bar{\zeta} \breve{L}$.

\section{The Free motion Schrödinger EQUATION ON THE LiNE}

Consider the case where $V:=\mathbb{R}$ is endowed with the Laplacian operator $L:=\partial^{2}$ on the smooth functions, namely on $A:=\mathcal{C}^{\infty}(\mathbb{R})$. The corresponding carré du champ is the usual one:

$$
\forall f, g \in A, \quad \Gamma[f, g]=2(\partial f)(\partial g) .
$$


Take $W:=\mathbb{R}_{+}$. To any $z \in W$, we associate the eigenvalue $\lambda_{z}=-z^{2}$, and a corresponding eigenvector $\varphi_{z}$ defined by

$$
\forall x \in \mathbb{R}, \quad \varphi_{z}(x):=\exp (i z x) .
$$

Note that the eigenvector $\varphi_{z}$ is only algebraic, in the sense that it satisfies the relation $L\left[\varphi_{z}\right]=\lambda_{z} \varphi_{z}$ everywhere on $\mathbb{R}$, but $\varphi_{z}$ does not belong to $\mathbb{L}^{2}$ space of the Lebesgue measure. For $z \in W \backslash\{0\}, z$ and $-z$ parametrize the same eigenvalue $-z^{2}$ and their eigenvectors $\varphi_{z}$ and $\varphi_{-z}=\overline{\varphi_{z}}$ are linearly independent. It appears that for any $f \in A$,

$$
\begin{aligned}
\forall x \in \mathbb{R}, \quad \check{L}_{z}[f](x) & =2 \exp (-i z x)(\partial \exp (i z x)) \partial f(x) \\
& =2 i z \partial f(x) .
\end{aligned}
$$

As in the end of the previous section, it leads us to take $\chi_{z}=1, \zeta_{z}=i$ and $\widehat{L}_{z}=2 z \partial$, the generator of the (deterministic) free motion at speed $2 z$. With the notation of Lemma 2.3, $\xi=i$ does not depend on $z \in W$.

Remark 3.1. As an illustration of Remark 2.1, we could have chosen $\xi=-i$, since the corresponding operator $\widehat{L}$ is the generator of the free motion at speed $-2 z$.

For any $z \in W$, the operators $L=\partial^{2}$ and $\widehat{L}_{z}$ commute and Remark 2.4 holds, with $B:=\mathbb{L}^{2}(\mu)$, where $\mu$ is the usual Lebesgue measure on $\mathbb{R}$, and with $C:=\mathbb{C}_{+}$and $\widehat{C}:=\mathbb{R}_{+}$. The assumptions of Section 2 are thus satisfied and we can apply Lemma 2.3. Consider a test function $f$ on $\mathbb{R}$. For any $(t, y, z) \in \mathfrak{V}$ and $s \in \mathbb{R}_{+}$, we have

$$
\mathfrak{P}_{s} R[f](t, y, z)=R P_{i s}[f](t, y, z) .
$$

The left-hand side is just $R[f](t+s, y+2 z s, z)$. Furthermore, from (2.5), we have for $t=0$,

$$
\begin{aligned}
\forall y \in \mathbb{R}, \forall z \in \mathbb{R}, \quad R[f](0, y, z) & =P_{0}[f](y) \\
& =f(y),
\end{aligned}
$$

so we deduce that

$$
\forall y \in \mathbb{R}, \forall z \in \mathbb{R}, \forall s \geqslant 0, \quad R[f](s, y+2 z s, z)=P_{i s}[f](y) .
$$

Thus, we have solved the free Schrödinger equation on the line, i.e. we have found an expression for the solution $u: \mathbb{R}_{+} \times \mathbb{R} \ni(s, y) \mapsto P_{i s}[f](y) \in \mathbb{C}$ of

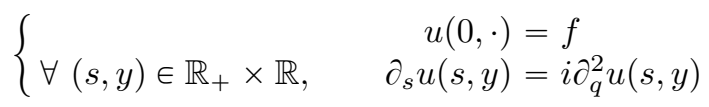

At least if we are able to identify directly the kernel $R$, i.e. without just inverting (3.3)! It follows from (2.5) that for any test function $f$ on $\mathbb{R}$,

$$
\forall(t, y, z) \in \mathfrak{V}, \quad R[f](t, y, z)=\frac{\exp \left(-i t \lambda_{z}\right)}{\exp (i z y)} P_{i t}[\exp (i z \cdot) f](y) .
$$

It may seem that we have not made much progress, since we still have to compute $P_{i t}[\varphi f](y)$. Indeed, let us complete this task, just as an illustration since our goal is to by-pass such computations. 
Recall that for any $s>0$ and $y \in \mathbb{R}$, we have the following expression for the heat kernel:

$$
P_{s}[f](y)=\int_{\mathbb{R}} f(x) \exp \left(-(x-y)^{2} /(4 s)\right) \frac{d x}{\sqrt{4 \pi s}} .
$$

By using the holomorphic extension of $\sqrt{\cdot}$ on $\mathbb{C} \backslash(-\infty, 0]$, the above formula is also valid for $s \in \mathbb{C} \backslash(-\infty, 0]$, for appropriate test functions (e.g. when $f$ is continuous and with compact support). It follows that

$$
\begin{aligned}
\forall(t, y, z) \in \mathfrak{V}, \quad R[f](t, y, z) & =\frac{\exp \left(-i t \lambda_{z}\right)}{\exp (i z y)} \int_{\mathbb{R}} f(x) \exp (i z x) \exp \left(-(x-y)^{2} /(4 i t)\right) \frac{d x}{\sqrt{4 \pi i t}} \\
& =\int_{\mathbb{R}} f(x) R(t, y, z ; x) d x,
\end{aligned}
$$

where

$$
R(t, y, z ; x):=\frac{1}{\sqrt{4 \pi i t}} \exp \left(i t z^{2}+i z(x-y)+i \frac{(x-y)^{2}}{4 t}\right) .
$$

Nevertheless, the main advantage of (3.4) is to suggest the introduction of appropriate "concentrating" mappings to avoid direct computations. More precisely, for any $h>0$ and $\left(y_{0}, z_{0}\right) \in \mathbb{R}^{2}$, consider the function $f_{h, y_{0}, z_{0}}$ given by

$$
\forall x \in \mathbb{R}, \quad f_{h, y_{0}, z_{0}}(x):=\exp \left(-i z_{0}\left(x-y_{0}\right)-\left(x-y_{0}\right)^{2} /(2 h)\right) /(\pi h)^{1 / 4} .
$$

Extending as usual the action of $P_{h}$ to probability measures, the function $f_{h, y_{0}, z_{0}}$ can be written as

$$
f_{h, y_{0}, z_{0}}=\sqrt{2}(\pi h)^{1 / 4} \frac{\varphi_{z_{0}}\left(y_{0}\right)}{\varphi_{z_{0}}} P_{h / 2}\left[\delta_{y_{0}}\right]
$$

We also have $\left|f_{h, y_{0}, z_{0}}\right|^{2}=P_{h / 4}\left[\delta_{y_{0}}\right]$ and this relation explains the choice of the normalization in (3.6). We deduce that for small $h>0,\left|f_{h, y_{0}, z_{0}}\right|^{2}$ is an approximation of the Dirac mass $\delta_{y_{0}}$, since it is the Gaussian density of mean $y_{0}$ and variance $h / 4$.

It follows from (3.4) (with $z$ replaced by $z_{0}$ ) and (3.7) that for all $t \geqslant 0$ and $x \in \mathbb{R}$,

$$
\begin{aligned}
R\left[f_{h, y_{0}, z_{0}}\right]\left(t, x, z_{0}\right) & =\sqrt{2}(\pi h)^{1 / 4} \frac{\exp \left(i t z_{0}^{2}\right) \varphi_{z_{0}}\left(y_{0}\right)}{\varphi_{z_{0}}(x)} P_{i t}\left[\frac{\varphi_{z_{0}}}{\varphi_{z_{0}}} P_{h / 2}\left[\delta_{y_{0}}\right]\right](x) \\
& =\sqrt{2}(\pi h)^{1 / 4} \frac{\exp \left(i t z_{0}^{2}\right) \varphi_{z_{0}}\left(y_{0}\right)}{\varphi_{z_{0}}(x)} P_{h / 2+i t}\left[\delta_{y_{0}}\right](x) .
\end{aligned}
$$

Namely, we have for any $t \geqslant 0$ and $x \in \mathbb{R}$,

$$
R\left[f_{h, y_{0}, z_{0}}\right]\left(t, x, z_{0}\right)=\left(\frac{h}{\pi}\right)^{1 / 4} \sqrt{\frac{1}{h+2 i t}} \exp \left(-i z_{0}\left(x-y_{0}\right)+i t z_{0}^{2}-\frac{\left(x-y_{0}\right)^{2}}{2(h+2 i t)}\right) .
$$

Normalizing the time $t$ into $h t$, we get

$$
R\left[f_{h, y_{0}, z_{0}}\right]\left(h t, x, z_{0}\right)=\left(\frac{1}{h \pi}\right)^{1 / 4} \sqrt{\frac{1}{(1+2 i t)}} \exp \left(-i z_{0}\left(x-y_{0}\right)+i h t z_{0}^{2}-\frac{\left(x-y_{0}\right)^{2}}{2 h(1+2 i t)}\right) .
$$


Finally we deduce from (3.3) that for $f_{h, y_{0}, z_{0}}$ given by (3.6),

$$
\begin{aligned}
& P_{i h t}\left[f_{h, y_{0}, z_{0}}\right](x) \\
& =R\left[f_{h, y_{0}, z_{0}}\right]\left(h t, x+2 z_{0} h t, z_{0}\right) \\
& =\left(\frac{1}{h \pi}\right)^{1 / 4} \frac{1}{\sqrt{1+2 i t}} \exp \left(-i z_{0}\left(x+2 z_{0} h t-y_{0}\right)+i h t z_{0}^{2}-\frac{\left(x+2 z_{0} h t-y_{0}\right)^{2}}{2 h(1+2 i t)}\right) \\
& =\left(\frac{1}{h \pi}\right)^{1 / 4} \frac{1}{\sqrt{1+2 i t}} \exp \left(-i z_{0}\left(x+2 z_{0} h t-y_{0}\right)+i h t z_{0}^{2}-(1-2 i t) \frac{\left(x+2 z_{0} h t-y_{0}\right)^{2}}{2 h \sqrt{1+4 t^{2}}}\right) .
\end{aligned}
$$

In particular, we get,

$$
\forall x \in \mathbb{R}, \quad\left|P_{i h t}\left[f_{h, y_{0}, z_{0}}\right](x)\right|^{2}=\frac{1}{\sqrt{\pi h\left(1+4 t^{2}\right)}} \exp \left(-\frac{\left(x+2 z_{0} h t-y_{0}\right)^{2}}{h \sqrt{1+4 t^{2}}}\right),
$$

which is the Gaussian density of mean $y_{0}-2 z_{0} h t$ and variance $h \sqrt{1+4 t^{2}} / 2$. This result is well-known and corresponds to the quantification of the free motion on $\mathbb{R}$ with speed $-2 z_{0}$, the mapping $\left|P_{i h t}\left[f_{h, y_{0}, z_{0}}\right]\right|^{2}$ being strongly concentrated around $y_{0}-2 z_{0} h t$ for $h>0$ small.

Of course, we could have computed directly $P_{i h t}\left[f_{h, y_{0}, z_{0}}\right](y)$, starting from the formulas (3.5) and (3.6), but the apparition of the free motion $\mathbb{R}_{+} \ni t \mapsto y_{0}-2 z_{0} t$ would (maybe) have been more mysterious. Furthermore, the intertwining relation (3.3) imposed the form of the concentrating mappings $f_{h, y_{0}, z_{0}}$.

Indeed, let us translate the above observations into the framework presented in the introduction, to show Theorem 1.5. On $\mathcal{V}:=\mathbb{R} \times \mathbb{R}$, consider the unitary algebra $\mathcal{A}$ of continuous functions $f$ such that for any fixed $z \in \mathbb{R}, f(\cdot, z) \in A$, namely $f$ is globally continuous and smooth in the first variable. Define on $\mathcal{A}$ the operator $\mathcal{L}$ given by

$$
\begin{aligned}
\forall f \in \mathcal{A}, \forall(y, z) \in \mathcal{V}, \quad \mathcal{L}[f](y, z) & :=\widehat{L}_{z}[f(\cdot, z)](y) \\
& =2 z \partial_{y} f(y, z) .
\end{aligned}
$$

It is the generator of the free motion: for any initial point $\left(y_{0}, z_{0}\right) \in \mathcal{V}$, the motion generated by $\mathcal{L}$ is given by

$$
\forall s \geqslant 0, \quad(Y(s), Z(s)):=\left(y_{0}+2 z_{0} s, z_{0}\right) .
$$

Consider the generator $\mathfrak{L}$ given by $(1.1)$, a corresponding Markov process starting from $\left(t_{0}, y_{0}, z_{0}\right)$ is $\mathfrak{X}:=(\mathfrak{X}(s))_{s \geqslant 0}:=\left(t_{0}+s, y_{0}+2 z_{0} s, z_{0}\right)_{s \geqslant 0}$.

As suggested by (2.6), define for any $h>0,(t, y, z) \in \mathfrak{V}, x \in V$,

$$
\begin{aligned}
F_{h}(t, y, z ; x) & :=R\left[f_{h, y, z}\right](t, x, z) \\
& =\left(\frac{h}{\pi}\right)^{1 / 4} \sqrt{\frac{1}{(h+2 i t)}} \exp \left(-i z(x-y)+i h t z^{2}-\frac{(x-y)^{2}}{2(h+2 i t)}\right) .
\end{aligned}
$$

Since we have

$$
\forall x \in \mathbb{R}, \quad\left|F_{h}(h t, y, z ; x)\right|^{2}=\frac{1}{\sqrt{\pi h\left(1+4 t^{2}\right)}} \exp \left(-\frac{(x-y)^{2}}{h \sqrt{1+4 t^{2}}}\right) .
$$


Hypotheses (H1) and (H2) are satisfied. In the latter, the concentration is even strong: for any $k \in \mathbb{Z}_{+}$, any $T \geqslant 0$ and any $r>0$,

$$
\lim _{h \rightarrow 0_{+}} \frac{1}{h^{k}} \sup _{(t, y, z) \in[0, T] \times \mathbb{R} \times \mathbb{R}} \int_{|x-y| \geqslant r}\left|F_{h}(h t, y, z ; x)\right|^{2} \mu(d x)=0 .
$$

Hypothesis (H3) is a direct consequence of (3.3), so Theorem 1.5 is shown.

Remark 3.2. In traditional semi-classical analysis, for $h>0$ and $\left(y_{0}, z_{0}\right) \in \mathbb{R}^{2}$, the functions $g_{h, y_{0}, z_{0}}:=f_{h, y_{0}, z_{0} / h}$ are often preferred to $f_{h, y_{0}, z_{0}}$ and lead to the following formulas valid for $x \in \mathbb{R}$,

$$
P_{i h t}\left[g_{h, y_{0}, z_{0}}\right](x)=\left(\frac{1}{h \pi}\right)^{1 / 4} \frac{1}{\sqrt{1+2 i t}} \exp \left(\frac{-i z_{0}\left(x+2 z_{0} t-y_{0}\right)+i t z_{0}^{2}}{h}-(1-2 i t) \frac{\left(x+2 z_{0} t-y_{0}\right)^{2}}{2 h \sqrt{1+4 t^{2}}}\right)
$$

and

$$
\left|P_{i h t}\left[g_{h, y_{0}, z_{0}}\right](x)\right|^{2}=\frac{1}{\sqrt{\pi h\left(1+4 t^{2}\right)}} \exp \left(-\frac{\left(x+2 z_{0} t-y_{0}\right)^{2}}{h \sqrt{1+4 t^{2}}}\right)
$$

It amounts to consider the process $(\mathfrak{X}(h s))_{s \geqslant 0}=(h s, Y(h s), Z(h s))_{s \geqslant 0}$ starting from $(0, y, z / h)$, and for this purpose, it is important that $z$ is not confined to a compact set in the supremum in (H2). In this way, we recover the classical motion

$$
\forall s \geqslant 0, \quad Y(h s)=y+2 z s .
$$

This high frequency normalization (leading to comparison of quantum times $h t$, in the above lhs, to classical times $t$, in the above rhs) does not seem so natural in our Markov process context, specially when the fibers $W$ are not vector spaces, as in the next sections.

Another manner to obtain the classical motion is to consider a small frequency normalization and long times: consider the process $(\mathfrak{X}(s / h))_{s \geqslant 0}=(s / h, Y(s / h), Z(s / h))_{s \geqslant 0}$ starting from $(0, y, h z)$, we get

$$
\forall s \geqslant 0, \quad Y(s / h)=y+2 z s .
$$

Unfortunately, this normalization is not very useful, since the concentration property (H2) holds only for times of order $h$.

We will see in the next sections to which extent this approach can be extended to discrete settings.

\section{THE FREE MOTION ON $\mathbb{Z}$}

We consider now $V:=\mathbb{Z}$. There are two natural difference operators on $\mathbb{Z}: \partial^{+}$and $\partial^{-}$, acting on $A$, the space of all bounded mappings from $\mathbb{Z}$ to $\mathbb{C}$, via

$$
\forall f \in A, \forall x \in \mathbb{Z}, \quad\left\{\begin{array}{l}
\partial^{+} f(x)=f(x+1)-f(x) \\
\partial^{-} f(x)=f(x-1)-f(x) .
\end{array}\right.
$$

It is immediate to check that

$$
\forall f \in A, \forall x \in \mathbb{Z}, \quad \partial^{+} \partial^{-} f(x)=\partial^{-} \partial^{+} f(x)=-\left(\partial^{+}+\partial^{-}\right) f(x)=2 f(x)-f(x+1)-f(x-1) .
$$


We endow $\mathbb{Z}$ with the discrete Laplacian $L=\partial^{+}+\partial^{-}$. The corresponding carré du champ is given by

$$
\begin{aligned}
\forall f, g \in A, \forall x \in \mathbb{Z}, \quad \Gamma[f, g](x)= & (f(x+1)-f(x))(g(x+1)-g(x)) \\
& +(f(x-1)-f(x))(g(x-1)-g(x)) .
\end{aligned}
$$

Take $W:=[0,2 \pi)$, seen as the set of angles of elements from $\mathbb{T}$, and for any $z \in W$, consider the function $\varphi_{z}$ defined by

$$
\forall x \in \mathbb{Z}, \quad \varphi_{z}(x):=\exp (i z x)
$$

It is an algebraic eigenvector of $L$ associated to the eigenvalue $\lambda_{z}=2(\cos (z)-1)$, but note that it does not belong to $l^{2}(\mathbb{Z})$. For $z \in(0, \pi), z$ and $z+\pi$ parametrize the same eigenvalue $2(\cos (z)-1)$ and their eigenvectors $\varphi_{z}$ and $\varphi_{-z}=\overline{\varphi_{z}}$ are linearly independent.

We compute that for any test function $f \in A$,

$$
\begin{aligned}
\forall x \in \mathbb{Z}, \quad \check{L}_{z}[f](x) & =\exp (-i z x) \Gamma[\exp (i z \cdot), f](x) \\
& =(\exp (i z)-1)(f(x+1)-f(x))+(\exp (-i z)-1)(f(x-1)-f(x)),
\end{aligned}
$$

namely

$$
\begin{aligned}
\check{L}_{z} & =(\exp (i z)-1) \partial^{+}+(\exp (-i z)-1) \partial^{-} \\
& =(\exp (-i z)-1) L+(\exp (i z)-1-(\exp (-i z)-1)) \partial^{+} \\
& =(\exp (-i z)-1) L+2 i \sin (z) \partial^{+} .
\end{aligned}
$$

So according to (2.7), for $z \in[0, \pi]$, we can take $\chi_{z}=\exp (-i z), \zeta_{z}=i$ and $\widehat{L}_{z}=2 \sin (z) \partial^{+}$, which is the generator of the Markov process always jumping toward the right, with intensity $2 \sin (z)$. These choices lead to $\xi_{z}=i \exp (-i z)$.

Remark 4.1. As another illustration of Remark 2.1, we could also have considered the decomposition

$$
\check{L}_{z}=(\exp (i z)-1) L-2 i \sin (z) \partial^{-},
$$

which leads to $\xi_{z}=-i \exp (i z)$, conjugate to its previous value.

For $z \in(\pi, 2 \pi)$, we proceed similarly, except that we rather take $\chi_{z}=\exp (i z), \zeta_{z}=i, \widehat{L}_{z}=-2 \sin (z) \partial^{-}$and $\xi_{z}=i \exp (i z)$. To simplify the presentation, from now on, we restrict $W$ to be $[0, \pi]$. The missing part $(\pi, 2 \pi)$ of the fibers can be treated similarly and enable to reverse the direction of the "free motion".

Due to the commutation properties mentioned at the beginning of this section, $L$ and $\widehat{L}_{z}$ commute. Note also that endowing $A$ with the supremum norm transforms it into a Banach algebra and that $L$ is bounded on $A$. The assumptions of Section 2 are satisfied and we can apply Lemma 2.3. Starting from $(0, y, z) \in \mathfrak{V}$, the operator $\mathfrak{L}$ generates the process $(s, Y(s), z)_{s \geqslant 0}$, where $(Y(s))_{s \geqslant 0}$ is a Markov process starting from $y$ and whose generator is $\widehat{L}_{z}$. More precisely, $(Y(s))_{s \geqslant 0}=(y+N(2 \sin (z) s))_{s \geqslant 0}$, where $(N(s))_{s \geqslant 0}$ is a standard Poisson process starting from 0 and of intensity 1 . It follows that for any test function $f \in A,(3.3)$ has to be replaced by

$$
\forall y \in \mathbb{Z}, \forall z \in \mathbb{R}, \forall s \geqslant 0, \quad \mathbb{E}[R[f](s, Y(s), z)]=P_{\xi_{z} s}[f](y),
$$

where the operator $R$ is given by $(2.5)$ :

$$
\forall(t, y, z) \in \mathfrak{V}, \forall f \in A, \quad R[f](t, y, z)=\frac{\exp (-2 i(\cos (z)-1) t)}{\exp (i z y)} P_{i t}[\exp (i z \cdot) f](y) .
$$


Thus, we have found a probabilistic representation of a modified free Schrödinger equation on the discrete line, i.e. of the solution $u: \mathbb{R}_{+} \times \mathbb{Z} \ni(s, y) \mapsto P_{\xi_{z} s}[f](y) \in \mathbb{C}$ of

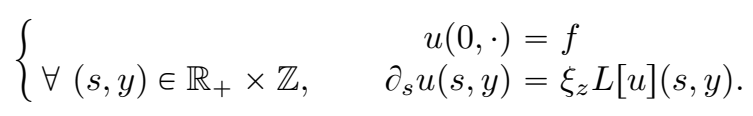

Contrary to the previous section, $\xi_{z} \in i \mathbb{R}$ only for $z \in\{0, \pi\}$, which corresponds to degenerate situations, since $\widehat{L}_{z}=0$ and so $Y(s)=y$ for all $s \geqslant 0$. This was to be predicted from Remark 1.1, asking in this situation for $(Y(s))_{s \geqslant 0}$ to be a deterministic process. For $z=0,(4.3)$ and (4.4) are both equivalent to $R[f](t, y, 0)=P_{i t}[f](y)$, for all $t \geqslant 0$ and $y \in \mathbb{Z}$. For $z=\pi,(4.3)$ and (4.4) are respectively equivalent to

$$
\forall t \geqslant 0, \forall y \in \mathbb{Z}, \quad R[f](t, y, \pi)=P_{-i t}[f](y),
$$

and

$$
\forall t \geqslant 0, \forall y \in \mathbb{Z}, \quad R[f](t, y, \pi)=\frac{\exp (4 i t)}{(-1)^{y}} P_{i t}\left[\varphi_{\pi} f\right](y)
$$

In view of Remark 1.4, another intriguing case is when $\xi_{z} \in\{ \pm 1\}$. Here, it corresponds to $z=\pi / 2$ and we get $\xi_{\pi / 2}=1, \varphi_{\pi / 2}(x)=i^{x}$ for all $x \in \mathbb{Z}$ and $\lambda_{\pi / 2}=-2$. In this situation we have

$$
\forall t \geqslant 0, \forall y \in \mathbb{Z}, \quad R[f](t, y, \pi / 2)=\frac{\exp (2 i t)}{i^{y}} P_{i t}\left[\varphi_{\pi / 2} f\right](y),
$$

and (4.3) leads to a strange formula: for any $f \in A$ and $y \in \mathbb{Z}$,

$$
\forall s \geqslant 0, \quad \mathbb{E}\left[\frac{\exp (2 i s)}{i^{y+N(2 s)}} P_{i s}\left[\varphi_{\pi / 2} f\right](y+N(2 s))\right]=P_{s}[f](y) .
$$

Let us now see how some features of the treatment of the free motion on $\mathbb{R}$ presented in the previous section can be adapted to the the present discrete setting of $\mathbb{Z}$.

Following the strategy described in (2.6), define for any $h>0$ and $(y, z) \in \mathbb{Z} \times[0, \pi]$,

$$
\forall x \in \mathbb{Z}, \quad f_{h, y, z}(x):=\delta_{y}(x),
$$

where the Kronecker delta appears in the rhs. Note that the function $f_{h, y, z}$ is so concentrated "around" $y$ that the parameters $h$ and $z$ do not play a role. Next we consider, for any $h>0,(t, y, z) \in \mathfrak{V}$ and $x \in V$,

$$
\begin{aligned}
F_{h}(t, y, z ; x) & :=R\left[f_{h, y, z}\right](t, x, z) \\
& =\frac{\exp \left(-i \lambda_{z} t\right)}{\varphi_{z}(x)} P_{i t}\left[\varphi_{z} f_{h, y, z}\right](x) \\
& =\frac{\exp \left(-i \lambda_{z} t\right) \varphi_{z}(y)}{\varphi_{z}(x)} P_{i t}\left[\delta_{y}\right](x) \\
& =\exp (-2 i(\cos (z)-1) t+i z(y-x)) g_{t, y}(x)
\end{aligned}
$$

where

$$
\forall t \geqslant 0, y \in \mathbb{Z}, \forall x \in \mathbb{Z}, \quad g_{t, y}(x):=P_{i t}\left[\delta_{y}\right](x) .
$$


Let $\mu$ the counting measure on $\mathbb{Z}$. The validity of (H1) and (H2) is provided by

Lemma 4.2. For any $t \geqslant 0$ and $y \in \mathbb{Z}$,

$$
\sum_{x \in \mathbb{Z}}\left|g_{t, y}(x)\right|^{2}=1
$$

and for any $T \geqslant 0$,

$$
\lim _{h \rightarrow 0_{+}} \frac{1}{h^{2}} \sup _{t \in[0, T], y \in \mathbb{Z}} \sum_{x \in \mathbb{Z} \backslash\{y\}}\left|g_{t, y}(x)\right|^{2}=2 .
$$

Proof. Since the operator $L$ is self-adjoint in $\mathbb{L}^{2}(\mu)$, the operator $P_{i h}$ is unitary in $\mathbb{L}^{2}(\mu)$. It follows that

$$
\begin{aligned}
\sum_{x \in \mathbb{Z}}\left|g_{t, y}(x)\right|^{2} & =\sum_{x \in \mathbb{Z}}\left|\delta_{y}(x)\right|^{2} \\
& =1,
\end{aligned}
$$

namely the first equality of the lemma is true. The second equality is equivalent to

$$
\lim _{h \rightarrow 0_{+}} \frac{1}{h^{2}} \sup _{t \in[0, T], y \in \mathbb{Z}}\left(1-\left|g_{t, y}(y)\right|^{2}\right)=2 .
$$

By definition of $P_{i h}=\exp (i h L)$ on the Banach algebra $A$, for any $y, x \in \mathbb{Z}$, we have the following expansion for $h>0$ small,

$$
P_{i h}\left[\delta_{y}\right](x)=\delta_{y}(x)+i h L\left[\delta_{y}\right](x)+\frac{(i h)^{2}}{2} L^{2}\left[\delta_{y}\right](x)+\circ\left(h^{2}\right)
$$

where the term $\circ\left(h^{2}\right)$ is uniform over $y, x \in \mathbb{Z}$. In particular, for $x=y$, we get

because

$$
g_{t, y}(y)=1-2 i h-3 h^{2}+\circ\left(h^{2}\right)
$$

$$
\begin{aligned}
L\left[\delta_{y}\right](y) & =L(y, y)=-2 \\
L^{2}\left[\delta_{y}\right](y) & =\sum_{y^{\prime} \in \mathbb{Z}} L\left(y, y^{\prime}\right) L\left(y^{\prime}, y\right) \\
& =L(y, y)^{2}+L(y, y+1) L(y+1, y)+L(y, y-1) L(y-1, y) \\
& =6 .
\end{aligned}
$$

We deduce that

$$
\begin{aligned}
\left|g_{t, y}(y)\right|^{2} & =\left|1-2 h i-3 h^{2}+\circ\left(h^{2}\right)\right|^{2} \\
& =\left(1-3 h^{2}\right)^{2}+(2 h)^{2}+\circ\left(h^{2}\right) \\
& =1-2 h^{2}+\circ\left(h^{2}\right),
\end{aligned}
$$

and the announced result follows. 
Hypothesis (H3) is a direct consequence of (4.3) applied with $f$ replaced by $f_{h, y, z}$, since

$$
\forall h>0, \forall(y, z) \in \mathbb{Z} \times[0, \pi], \forall x \in \mathbb{Z}, \quad F_{h}(0, y, z ; x)=f_{h, y, z}(x),
$$

and since under $\mathbb{P}_{(0, y, z)}$,

$$
\forall s \geqslant 0, \quad \mathfrak{X}(s)=(s, y+N(2 \sin (z) s), z) .
$$

Remark 4.3. In the spirit of Remark 3.2, it is now only possible to consider the not very convincing small frequency normalization: replacing in (4.8) $s$ by $s / h$ and $z$ by $z h$ (assuming $z \in[0, \pi / h]$ ), as $h$ goes to $0_{+}$, the base component converges toward the process $(y+N(2 z s))_{s \geqslant 0}$, which is a Poisson process whose rate $2 z$ can be as high as wanted (for $h$ small).

Remark 4.4. It is tempting to play with the objects at hand, to see how the results are affected by their modifications. For instance, we could replace the operator $\widetilde{L}$ defined in $(2.2)$ by $(1-\epsilon) \widetilde{L}+\epsilon L$, where $\epsilon \in(0,1)$. Then we have

$$
\widetilde{L}^{(\epsilon)}=\chi^{(\epsilon)} L+\zeta \widehat{L}^{(\epsilon)}
$$

with

$$
\begin{aligned}
\widetilde{L}^{(\epsilon)} & :=\frac{(1-\epsilon) \widetilde{L}+\epsilon L}{a_{\epsilon}} \\
a_{\epsilon} & :=|\epsilon+(1-\epsilon) \chi| \\
\chi^{(\epsilon)} & :=\frac{\epsilon+(1-\epsilon) \chi}{a_{\epsilon}} \in \mathbb{T} \\
\widehat{L}^{(\epsilon)} & :=\frac{1}{a_{\epsilon}} \widehat{L} .
\end{aligned}
$$

It follows that $\xi^{(\epsilon)}=\xi(\epsilon \bar{\chi}+(1-\epsilon)) / a_{\epsilon}$. In the setting of the present section, we get for $z \in[0, \pi]$,

$$
\xi_{z}^{(\epsilon)}=\xi_{z}(\epsilon \exp (i z)+(1-\epsilon)) /|\epsilon \exp (i z)+(1-\epsilon)|,
$$

and this complex number can be "more imaginary" than $\xi_{z}$. Remark 1.4 may then let us believe that is advantageous to consider such transformations with $\epsilon \in(0,1)$. But it is wrong, because computing the corresponding operator $R^{(\epsilon)}$ and the functions $\left(F_{h}^{(\epsilon)}\right)_{h>0}$ (via (2.6) with the functions $f_{h, y, z}$ given by (4.5)), we get that (H2) is not satisfied, the concentration being only of order 0 .

\section{THE FREE MOTIONS ON TORI}

After proving Theorems 1.7, 1.8 and 1.9 in their respective torus settings, we will discuss generally about the tensorization of multi-dimensional quantification.

The case of $V=\mathbb{Z}_{n}$, for a given $n \in \mathbb{N} \backslash\{1\}$, is very similar to the situation of $\mathbb{Z}$ described in the previous section. The difference operators $\partial^{-}$and $\partial^{+}$are extended to act on $\mathbb{Z}_{n}$, which is endowed with the discrete Laplacian $L:=\partial^{-} \partial^{+}=\partial^{+} \partial^{-}=\partial^{-}+\partial^{+}$(when $n=2$, we have furthermore $\partial^{-}=\partial^{+}$and $L=2 \partial^{+}$). The underlying Banach algebra $A_{n}$ is just the usual algebra of all $\mathbb{C}$-valued functions defined on $\mathbb{Z}_{n}$. The carré du champs of $L$ is still given by (4.1), where $x$ takes values in $\mathbb{Z}_{n}$. Let $W:=W_{n}:=\frac{2 \pi}{n} \llbracket 0, n-1 \rrbracket$ and consider for any $z \in W_{n}$, the function

$$
\forall x \in \mathbb{Z}_{n}, \quad \varphi_{z}(x):=\exp (i z x),
$$


which is an eigenvector of $L$ associated to the eigenvalue $\lambda_{z}:=2(\cos (z)-1)$. All the computations and observations of the previous section are still valid, once $\mathbb{Z}$ has been replaced by $\mathbb{Z}_{n}$ and $[0,2 \pi)$ by $W_{n}$. In particular, for $z \in W_{n} \cap[0, \pi]=(2 \pi / n) \llbracket 0,\lfloor n / 2] \rrbracket$ (where $\lfloor\cdot\rfloor$ stands for the integer part), we can choose $\widehat{L}_{z}=2 \sin (z) \partial^{+}$, with $\chi_{z}=\exp (-i z), \zeta_{z}=i$ and $\xi_{z}=i \exp (-i z)$. Again we can apply Lemma 2.3: starting from $(0, y, z) \in \mathfrak{V}=\mathbb{R}_{+} \times \mathbb{Z}_{n} \times W_{n}$, the operator $\mathfrak{L}$ generates the process $(s, Y(s), z)_{s \geqslant 0}$, where $(Y(s))_{s \geqslant 0}$ is a Markov process starting from $y$ and whose generator is $\widehat{L}_{z}$. Namely, $(Y(s))_{s \geqslant 0}=(y+N(2 \sin (z) s)[n])_{s \geqslant 0}$, where $(N(s))_{s \geqslant 0}$ is a standard Poisson process starting from 0 and of intensity 1 , and where $[n]$ means modulo $n$. In conformity with $(1.1), \mathcal{L}:=\widehat{L}_{z}$ is the generator of the process $(Y(s), z)_{s \geqslant 0}$. Going through the same constructions of $R,\left(f_{h, y, z}\right)_{h>0, y \in \mathbb{Z}_{n}, z \in W_{n}}$ and $\left(F_{h}\right)_{h>0}$, given respectively in (4.4), (4.5) and (2.6) (see also (4.6)), we conclude to the validity of Theorem 1.7.

Remark 5.1. Contrary to Remarks 3.2 and 4.3, neither the high frequency nor the fruitless small frequency normalizations are possible for the above quantification, since $W_{n}$ is finite.

The case of $V=\mathbb{R} /(2 \pi \mathbb{Z})$, has similarities with both the situations of $\mathbb{R}$ and $\mathbb{Z}_{n}$. We consider the Laplacian operator $L=\partial^{2}$ on the algebra $A$ of smooth functions defined on $\mathbb{R} /(2 \pi \mathbb{Z})$. Its carré du champs is given by (3.1). Take $W=\mathbb{Z}$, to any $z \in \mathbb{Z}$, we associate the eigenvalue $\lambda_{z}=-z^{2}$ and a corresponding eigenvector $\varphi_{z}$ is defined by

$$
\forall x \in \mathbb{R} /(2 \pi \mathbb{Z}), \quad \varphi_{z}(x):=\exp (i z x) .
$$

As in Section 3, for any $z \in \mathbb{Z}$, we take $\chi_{z}=1, \zeta_{z}=i, \xi_{z}=i$ and $\widehat{L}_{z}=2 z \partial$. Lemma 2.3 can be applied: starting from $(0, y, z) \in \mathfrak{V}=\mathbb{R}_{+} \times \mathbb{R} /(2 \pi \mathbb{Z}) \times \mathbb{Z}$, the operator $\mathfrak{L}$ generates the process $(\mathfrak{X}(s))_{s \geqslant 0}=(s, y+2 z s, z)_{s \geqslant 0}$. In conformity with $(1.1), \mathcal{L}:=\widehat{L}_{z}$ is the generator of the process $(y+2 z s, z)_{s \geqslant 0}$. Going through the same constructions of $R,\left(f_{h, y, z}\right)_{h>0, y \in \mathbb{R} /(2 \pi \mathbb{Z}), z \in \mathbb{Z}}$ and $\left(F_{h}\right)_{h>0}$, given respectively in (3.4), (3.7) (in both equations, $\left(P_{t}\right)_{t \geqslant 0}$ is now the heat semi-group generated by $L$ on $\left.\mathbb{R} /(2 \pi \mathbb{Z})\right)$ and $(2.6)$, we conclude to the validity of Theorem 1.9.

Remark 5.2. Similarly to the first part of Remark 3.2 , it is possible to consider a high frequency normalization for the above quantification. More precisely, for given $z \in \mathbb{R}$, consider $H_{z}:=\{h>0: z / h \in \mathbb{Z}\}$. We get that for $h \in H_{z}$, the base component of the process $(\mathfrak{X}(h s))_{s \geqslant 0}=(h s, Y(h s), Z(h s))_{s \geqslant 0}$ starting from $(0, y, z / h)$ is equal to $(y+2 z s)_{s \geqslant 0}$, the classical free motion on $\mathbb{R} /(2 \pi \mathbb{Z})$.

We now come to the situation of the free motions on finite multidimensional tori. With the notations introduced before Theorem 1.8, consider the Laplacian operator $L:=\sum_{j \in \llbracket m \rrbracket} L^{(j)}$, on the space $A$ of all $\mathbb{C}$-valued functions defined on $V$. For any $z:=\left(z_{1}, z_{2}, \ldots, z_{m}\right) \in W, \lambda_{z}:=2 \sum_{j \in \llbracket m \rrbracket}\left(\cos \left(z_{j}\right)-1\right)$ is an eigenvector of $L$ associated to the eigenfunction $\varphi_{z}$ given by

$$
\forall x:=\left(x_{1}, x_{2}, \ldots, x_{m}\right) \in V, \quad \varphi_{z}(x):=\exp \left(i \sum_{j \in \llbracket m \rrbracket} z_{j} x_{j}\right) .
$$

Considering the associated Doob transform $\widetilde{L}_{z}$ defined as in (2.1), (2.2) must be replaced by

$$
\widetilde{L}_{z}=\sum_{j \in \llbracket m \rrbracket} \chi_{z_{j}} L^{(j)}+i \widehat{L}_{z}
$$

where

$$
\forall j \in \llbracket m \rrbracket, \quad \chi_{z_{j}}:=\exp \left(-i z_{j}\right)
$$




$$
\begin{aligned}
\widehat{L}_{z} & :=2 \sum_{j \in \llbracket m \rrbracket}\left|\sin \left(z_{j}\right)\right| \partial^{(j), \varepsilon_{j}} \\
\forall j \in \llbracket m \rrbracket, \quad \varepsilon_{j} & :=\operatorname{sign}\left(\sin \left(z_{j}\right)\right) \\
\forall f \in A, \forall x \in V, \forall j \in \llbracket m \rrbracket, \forall \varepsilon \in\{-1,1\}, \quad \partial^{(j), \varepsilon} f(x) & :=f\left(x+\varepsilon e_{j}\right)-f(x) .
\end{aligned}
$$

Taking into account that the operators $L^{(1)}, L^{(2)}, \ldots, L^{(m)}$ and $L_{z}$ all commute, the computations of Section 2 can be extended to this situation. Consider $\mathfrak{L}$ the operator given in $(2.3)$ and replace the definition of the operator $R$ from $\mathfrak{V}$ to $V$ given in (2.5) by

$$
\begin{aligned}
\forall(t, y, z) \in \mathfrak{V}, \forall f \in A & , \\
R[f](t, y, z) & :=\frac{\exp \left(2 t \sum_{j \in \llbracket m \rrbracket}\left(\cos \left(z_{j}\right)-1\right) \bar{\zeta}_{z_{j}}\right)}{\varphi_{z}(y)} \exp \left(-t \sum_{j \in \llbracket m \rrbracket} \bar{\zeta}_{z_{j}} L^{(j)}\right)\left[\varphi_{z} f\right](y) .
\end{aligned}
$$

Lemma 2.3 has to be modified:

Lemma 5.3. We have

$$
\forall s \in \mathbb{R}_{+}, \quad \exp (s \mathfrak{L}) R=R \exp \left(s \sum_{j \in \llbracket m \rrbracket} \xi_{z_{j}} L^{(j)}\right),
$$

with $\xi_{z_{j}}:=i \exp \left(-i z_{j}\right)$, for all $j \in \llbracket m \rrbracket$.

This relation is equivalent to the generator intertwining

$$
\mathfrak{L} R=R \sum_{j \in \llbracket m \rrbracket} \xi_{z_{j}} L^{(j)} .
$$

The proof of Theorem 1.9 now follows the same track as before: the mappings $\left(f_{h, y, z}\right)_{h>0, y \in V, z \in W}$ and $\left(F_{h}\right)_{h>0}$ being defined respectively in (4.5) and (2.6).

The above presentation shows that the method based on the Doob transform with respect to complex valued eigenvectors can be applied in multi-dimensional settings. It would be interesting to investigate relations such as (5.2) in more general situation, when the operators entering in the decomposition are not commuting, even in one dimension.

Nevertheless, note that multi-dimensional quantification can be directly tensorized, without going through the Doob transform arguments. In the framework of the introduction, consider two families $\left(L_{l}^{(j)}\right)_{j \in J_{l}}$, for $l \in\{1,2\}$ and two disjoint finite index sets $J_{1}$ and $J_{2}$, of reversible Markov generators on $\mathbb{L}^{2}\left(V_{l}, \mu_{l}, \mathbb{C}\right)$, where $V_{l}$ is the state space endowed with the measure $\mu_{l}$. Assume that they are respectively the multidimensional quantifications of operators $\mathcal{L}_{l}$, whose state space is $\mathcal{V}_{l}$, in the sense that (H1), (H2) and (H4) are satisfied. Define

$$
\begin{aligned}
V & :=V_{1} \times V_{2} \\
\mu & :=\mu_{1} \otimes \mu_{2} \\
J & :=J_{1} \sqcup J_{2} \\
\forall j \in J, \quad L^{(j)} & :=\left\{\begin{array}{c}
L_{1}^{(j)}, \text { if } j \in J_{1} \\
L_{2}^{(j)}, \text { if } j \in J_{2}
\end{array}\right. \\
\mathcal{V} & :=\mathcal{V}_{1} \times \mathcal{V}_{2} \\
\mathcal{L} & :=\mathcal{L}_{1}+\mathcal{L}_{2},
\end{aligned}
$$


where $\mathcal{L}_{1}$ (respectively $\mathcal{L}_{2}$ ) acts on the first (resp. second) component of $\mathcal{V}$. We get then.

Proposition 5.4. The family $\left(L^{(j)}\right)_{j \in J}$ is the multi-dimensional quantification of $\mathcal{L}$.

Proof. For $l \in\{1,2\}$, let $\left(F_{l, h}\right)_{h>0}$ be a family of mappings such that (H1), (H2) and (H4) are satisfied, for the multidimensional quantification of the operator $\mathcal{L}_{l}$ by $\left(L_{l}^{(j)}\right)_{j \in J_{l}}$. Define the family $\left(F_{h}\right)_{h>0}$ by tensorization of these families: for any $h>0$,

$$
\forall t \geqslant 0, \forall\left(y_{1}, z_{1}\right) \in \mathcal{V}_{1}, \forall\left(y_{2}, z_{2}\right) \in \mathcal{V}_{2}, \quad F_{h}\left(t,\left(y_{1}, y_{2}\right),\left(z_{1}, z_{2}\right)\right):=F_{1, h}\left(t, y_{1}, z_{1}\right) F_{2, h}\left(t, y_{2}, z_{2}\right) .
$$

Hypotheses (H1) and (H2) are clearly satisfied, once $V$ is endowed with the distance $\rho$ given, e.g., by

$$
\forall\left(x_{1}, x_{2}\right),\left(y_{1}, y_{2}\right) \in V, \quad \rho\left(\left(x_{1}, x_{2}\right),\left(y_{1}, y_{2}\right)\right):=\max \left(\rho_{1}\left(x_{1}, y_{1}\right), \rho_{2}\left(x_{2}, y_{2}\right)\right),
$$

where $\rho_{1}$ and $\rho_{2}$ are the underlying distance on $V_{1}$ and $V_{2}$. Hypothesis (H4) is also true, because a process $\mathcal{X}:=(s, Y(s), Z(s))_{s \geqslant 0}$, associated to $\mathfrak{L}=\partial_{s}+\mathcal{L}$ and starting from a point of $\mathfrak{V}$, can be written as $\left(s, Y_{1}(s), Y_{2}(s), Z_{1}(s), Z_{2}(s)\right)_{s \geqslant 0}$ where $\mathfrak{X}_{1}:=\left(s, Y_{1}(s), Z_{1}(s)\right)_{s \geqslant 0}$ and $\mathfrak{X}_{2}:=\left(s, Y_{2}(s), Z_{2}(s)\right)_{s \geqslant 0}$ are independent Markov processes respectively generated by $\mathfrak{L}_{1}=\partial_{s}+\mathcal{L}_{1}$ and $\mathfrak{L}_{2}=\partial_{s}+\mathcal{L}_{2}$ (in the sense of martingale problems). One should take in (H4), for any $j \in J, \xi_{0}^{(j)}:=\xi_{l, 0}^{(j)}$ when $j \in J_{l}$, and $\xi_{0}^{(j)}$ depends only on $\left(y_{l, 0}, z_{l, 0}\right)$, when $\left(y_{0}, z_{0}\right)=\left(y_{1,0}, y_{2,0}, z_{1,0}, z_{2,0}\right)$, with obvious notations.

Of course, the above construction can be extended to any finite number of factor spaces. In particular, we can take factor spaces coming from any of the examples given in Theorems 1.5, 1.6, 1.7 or 1.9. Theorem 1.8 has only taken factor spaces coming from Theorem 1.7. Note that when the factors all come from Theorems 1.5 and 1.9 , there is no need for the notion of multi-dimensional quantification, since quantification is sufficient. Indeed the product satisfies (H3), with $L:=\sum_{j \in J} L^{(j)}$, as we have

$$
\sum_{j \in J} \xi_{0}^{(j)} L^{(j)}=i L
$$

Acknowledgements. I'm grateful to Jean-Marc Bouclet for our discussions about the traditional quantification of the free motion on Euclidean spaces, to Francesco Costantino for the organization of a motivating working group on quantification at the IMT and to Sylvain Ervedoza for pointing out some references about discrete wave equations. I'm also indebted to the ANR STAB (Stabilité du comportement asymptotique d'EDP, de processus stochastiques et de leurs discrétisations : 12-BS01-0019) for its support.

\section{REFERENCES}

[1] D. Bakry, I. Gentil and M. Ledoux, Analysis and geometry of Markov diffusion operators. Vol. 348 of Grundlehren der Mathematischen Wissenschaften [Fundamental Principles of Mathematical Sciences]. Springer, Cham (2014).

[2] J. Giannoulis, M. Herrmann and A. Mielke, Continuum descriptions for the dynamics in discrete lattices: derivation and justification, in Analysis, Modeling and Simulation of Multiscale Problems. Springer, Berlin (2006) 435-466.

[3] F. Macià, Propagación y control de vibraciones en medios discretos y continuos. Ph.D. thesis, Universidad Complutense de Madrid, Departamento de Matemática Aplicada, Universidad Complutense de Madrid (2002).

[4] F. Macià, Wigner measures in the discrete setting: high-frequency analysis of sampling and reconstruction operators. SIAM J. Math. Anal. 36 (2004) 347-383.

[5] A. Mielke, Macroscopic behavior of microscopic oscillations in harmonic lattices via Wigner-Husimi transforms. Arch. Ration. Mech. Anal. 181 (2006) 401-448.

[6] M. Zworski, Semiclassical Analysis. Vol. 138 of Graduate Studies in Mathematics. American Mathematical Society, Providence, RI (2012). 